\title{
Image Edge Detection Algorithm Based on The Parallel Genetic and Otsu dual-threshold
}

\author{
Dang xiangying ${ }^{1,2, a}$, Li dan ${ }^{1,2}$ \\ ${ }^{1}$ Xuzhou Institute of Technology, Xuzhou, China \\ ${ }^{2}$ Xuzhou Machine Vision Application Engineering Technology Research Center, Xuzhou, China \\ ${ }^{a}$ E-mail: dangpaper@163.com
}

Keywords: Parallel; genetic algorithm; edge detection ;Otsu dual-threshold

\begin{abstract}
The traditional genetic algorithm is used to search some function extreme value, but it has low precision and poor stability. For this shortage, A kind of parallel genetic algorithm based on Otsu double threshold algorithm of image edge detection, the design into a line of two columns of the vector population, than as an individual's fitness variance between, generated a number of initial population of parallel computing, At the same time, the largest fitness individuals in each generation directly copied into the next generation, evolution in a number of different subgroup in parallel, in order to avoid the premature convergence phenomenon appeared in the process of single population evolution, to improve the algorithm convergence speed. The experimental results show that the algorithm is compared with the traditional genetic algorithm, can more accurate and has better accuracy and stability.
\end{abstract}

\section{Introduction}

The edge is an important feature of image target detection, the image edge detection is a basic in the field of image processing and computer vision problems, and which is the premise of image subsequent processing. The edge detection can deal with the many complicated problems .How to find the real target image edge, and it has always been a hot research topic in the image processing field, the traditional edge detection methods have difference operator method (Roberts, Sobel, Prewitt, Kirsch and Laplacian, etc.)、 template matching optimal curved surface fitting, etc. [1].These algorithms have made some achievements in improving edge detection effect, However, they have complicated mathematical model and a longer run time also.

Threshold value is a key method in the image edge detection algorithm, They are widely used because of simple, rapid and traditional Otsu method is a kind of the earliest by Otsu [2] in 1979, the classical threshold method is put forward, it is very effective and widely used [3]. This method uses one dimensional image gray histogram of the, make the exhaustive search pixels can be divided into two types of target and background, and the maximum variance between one dimensional threshold. Two-dimensional Otsu method [4-5] than traditional Otsu method has better noise performance.

In practical applications, the threshold value method is usually as a basic segmentation technology which can obtain good image segmentation effect. But the threshold method only consider pixel gray value in the process of image segmentation, without considering the space characteristics, so sensitive to noise.

The reason that the Otsu dual-threshold threshold is used, is the several threshold of the gray-level histogram can be divided into separate classes, making all kinds of the variance between the biggest. To determine the threshold value is the key of threshold segmentation. According to the number of threshold value, the image threshold can be divided into single threshold segmentation and the multiple threshold segmentation problem which can be converted into a series of single threshold segmentation problem to solve, but it will search a best threshold combination in the range of gray level, which encounter much more time consuming, difficult to practical application. To simplify the calculation, the available genetic and the immune evolutionary algorithm[6-7] which were used search the optimal threshold. Although the algorithm improves the speed of segmentation, the Otsu dual-threshold algorithm search result is not stable, thus get the threshold is not stable and accurate, lead to different image segmentation results. 
Because of these weaknesses, this paper designed image edge detection method combining with the OTSU threshold technology and the parallel genetic algorithm, on the one hand, using the parallel genetic algorithm to the high speed of parallel computer parallelism and parallel genetic algorithm, the combination of greatly improved genetic algorithm to solve the speed and quality, On the other hand, different subgroups are evolving in multiple parallel, the single population evolution phenomenon of premature convergence was avoided in the process, improve the rate of convergence of the algorithm. Simulation results show that the algorithm can accurately and quickly find the best threshold of image segmentation, and carries on the double threshold segmentation of image, through the best threshold edge detection and the result can be seen that the algorithm to improve the stability of algorithms in image edge detection and precision.

\section{The OTSU Threshold Method}

A grayscale range in the image for $\{0,1, \ldots, \mathrm{L}-1\}$, the threshold value is set $t$, which will be divided into two categories, the target image and the background image, the gray level' probability is set $P_{i}$, the probability of target parts is $\varpi_{0}=\sum_{i=0}^{t} p_{i}$, the probability of the part background of is $\varpi_{1}=\sum_{i=t+1}^{L-1} p_{i}$, the mean of the target is $\mu_{0}=\sum_{i=0}^{t} p_{i} * i / \varpi_{0}$, the mean of the background is $\mu_{1}=\sum_{i=t+1}^{L-1} p_{i} * i / \varpi_{1}$. The variance formula between the two groups is $\sigma^{2}=\varpi_{0}\left(\mu_{0}-\mu\right)^{2}+\varpi_{1}\left(\mu_{1}-\mu\right)^{2}$,as the best threshold, the optimal threshold is that take the maximum variance. Extending the Otsu method to double threshold segmentation, the optimal threshold value $t_{1}, t_{2}$, and they should get the maximum variance [1], that is

$$
\begin{gathered}
\sigma^{2}=\varpi_{0}\left(\mu_{0}-\mu\right)^{2}+ \\
\varpi_{1}\left(\mu_{1}-\mu\right)^{2}+\varpi_{2}\left(\mu_{2}-\mu\right)^{2} \\
: \mu=\sum_{i=0}^{L-1} p_{i}^{*}{ }^{t_{0}}, \quad \mu_{0}=\sum_{i=0}^{t_{0}} p_{i}^{*} i / \varpi_{0}, \\
\mu_{1}=\sum_{i=t_{1}+1}^{t_{2}} p_{i}^{*} i / \varpi_{1}, \mu_{2}=\sum_{i=t_{2}+1}^{L-1} p_{i}^{*} i / \varpi_{2}, \\
\varpi_{0}=\sum_{i=0}^{t_{1}} p_{i}, \quad \varpi_{1}=\sum_{i=t+1}^{t_{2}} p_{i}, \quad \varpi_{2}=\sum_{i=t_{2}+1}^{L-1} p_{i} .
\end{gathered}
$$

In this way, using the between-cluster variance method, the double threshold image segmentation threshold of solving problem can be summarized as the best threshold value, the optimization problem.

\section{An OTSU Dual-threshold based on Parallel Genetic Algorithm}

To seek more threshold algorithm complexity will be increased with the increase of number of segmentation threshold increased rapidly, operation time overhead than single threshold algorithm into geometric progression growth. The genetic algorithm (GA) is mimic natural biological evolution mechanism of the random global search and optimization method, it is a kind of efficient, parallel and global searching method, we can adaptively control the searching process to ensure the optimal solution. The optimal value of genetic algorithm in many areas has been widely used on the issue.

This paper uses the parallel genetic algorithm [8]to solve the image threshold segmentation, image segmentation algorithms are optimized.

First of all, The main processor distribute each population in different independent concurrent evolution operation on the main loop, then every population run certain algebra, each processor running from the main loop of genetic algorithm will receive upstream the main loop of the next best chromosomes, and downstream adjacent main loop transfer itself to the best chromosome. So it run 
down until termination condition is met. When at the end of the algorithm, each one from the processor best chromosomes, from processors will be its own best chromosomes to main processor, a main processor with optimal chromosome as the final result.

From the formula (1), we can know the double threshold image segmentation threshold of the solving problem is an optimization problem, combining with the characteristics of parallel genetic algorithm and put forward a kind of parallel genetic algorithm based on OTSU double threshold image segmentation transportation.

(1) The population initialization

In search space , $\mathrm{N}$ individuals were random produced as the initial population, and the fitness of each individual was calculated, the largest breeding algebra for NG, the initial values of the individuals in the population is randomly generated, its corresponding fitness value also have high and low, the i generation of each population is operated

(2) Coding scheme

Due to the image grey value between $0 \sim 255$, placing each dye to color coding for 16-bit binary code, the former eight represents another threshold segmentation, after eight represents another threshold segmentation.

(3) Fitness function

Using the formula (1) as a fitness function. According to the calculated sorting of individual fitness value, the most to adapt to the individual order is 1 , time to adapt to the individual order is 2 and so on.

(4) Selection method

Method used to bet on wheels.

(5) Cross

With double cross points, two intersection was done the former eight and the after eight, random generation, individual parts of the cross of random matching string after the intersection to swap.

(6) Mutation

According to the mutation probability randomly change the former eight and eight after a string of bits in the value (that is, the not).

(7) Termination criteria

When algorithm performs is maximum generation (termination conditions), the algorithm stop running, with the highest fitness value of individual is the segmentation threshold, Otherwise, the algorithm is to continue.

\section{The Experiment Result and Analysis}

The algorithm experiment tool by the Matlab simulation in this paper, for the convenience of display pages, choose standard tests in the library of 256*256 gray image Lena. The parameters of the parallel genetic algorithm is as follows: the number of processors is 2 , each processor handle a total of 10 populations of individuals, crossover probability is 0.8 , the mutation probability is 0.3 , iterative algebra for 200.On the two algorithms respectively independent experiment 50 times. As shown in Fig. 1 is the original image, as shown in the Fig. 2 is the traditional image edge detection base the genetic threshold methods of results, this paper calculated 100 times the average threshold and integer, the result of image edge detection in the Fig. 3 based on the OTSU double threshold of parallel genetic algorithms of image edge detection results.

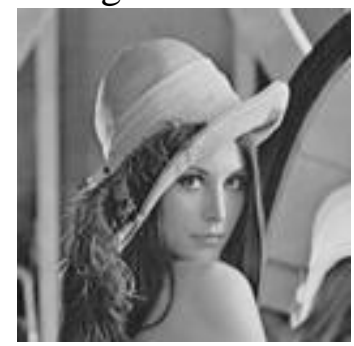

Fig.1 Lena original image

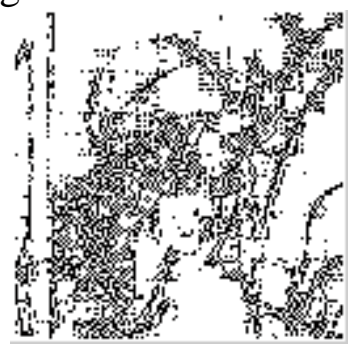

Fig.2 The traditional methods

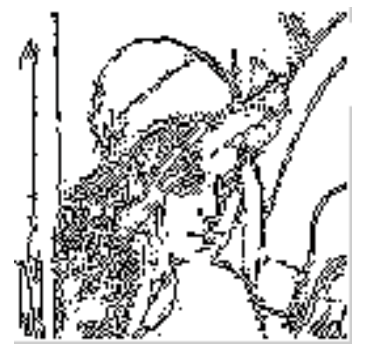

Fig.3 The proposed method

By contrast, we can be found in Fig. 2, the traditional threshold edge detection effect is not ideal, 
more rich details, but also produce a lot of false edge, the whole image noise is larger. Fig. 3 show the less false edge around the image edge, positioning precision, less noise, get the ideal image edge.

\section{Conclusion}

The contrast experiment shows that the parallel genetic algorithm proposed in this paper combining Otsu dual-threshold is successfully applied to image edge detection, not only keep the parallel genetic algorithm than the characteristics of the genetic algorithm convergence speed, under the condition of without increasing computation complexity, the optimal threshold search performance showed certain advantages, improve the accuracy and stability of the search.

\section{References}

[1] Y.Tian, F.Y.Peng.”Digital image processing and analysis”, Huazhong university of science and technology press ,Wuhan,pp. 122-134,2009.

[2] Otsu N. “ A threshold selection method from gray -level his tograms”. IEEE Transactions on System Man and Cybernetic, Vol.9 ,1979,pp. 62- 66.

[3] P.K.Sahoo,S.Soltani.”A survey of thresholding techniques”Computer Vision, Graphics and Image Processing, Vol.41, 1988, pp.233-260.

[4] J.Z.Liu,W.Q.Su. Au tomatic thres holding of gray level pictures using two - dimension Otsu method, Acta Auto matica Sinica, Vol.19,1993, pp.101- 105.

[5] Z.Y.He, L.N.Sun. "Fast Computation of Threshold Based on Otsu Criterion”, Acta Electronica Sinica, Vol.41,2013,pp.267-272.

[6] C.Jin,J.X.Peng, "Image Gray Multi-Level Threshold Selection Method Based on Genetic Strategy”. Computer Engineering And Application, vol. 39, 2003,pp.23-26.

[7] J.X.Huang,H.L.” A Threshold Selection Method of I mage Segmentation Based on Genetic Algorithms”,Journal of NanJing Normal University. Vol . 7,14-17,36.

[8] T.C.Guo,C.D.Mu. “The Parallel Drift s of Genetic Algorithms”, Systems engineering theory and practice, VOL.2, 2002,pp. 15-23. 